\title{
Develop a TB88 step-up circuit for minor thermoelectric signal
}

\begin{abstract}
A TB88 step-up circuit for minor thermoelectric signal, induced by chip "TEG12703 ", is developed in this study. Here several hundred microvolts could be created if finite differential temperature on the side surface of thermo electric chip is imposed. Such small amount of voltage induced seems to be not adequate to meet the demand of commercial benefit unless a step-up circuit to augment the electrical potential is specified. In this article, a step-up circuit of TB88 IC with thermoelectric-electricity transforming efficiency $55 \sim 65 \%$ has been carried out and successfully boosted the voltage from $0.5 \mathrm{~V}$ to $5 \mathrm{~V}$. That not only holds an extra $40 \%$ advantage of power delivery, compared to the performance accessed from commercial amplified device " CE8301" (Figure 1), but also significantly reduces the experimental cost required and easily accessed for local laboratory.
\end{abstract}

Volume 2 Issue 3 - 2017

Je-Ee Ho, Shih-Syun Chen, Yu-Min Hung Department of Mechanical Engineering, National Ilan University, Taiwan

Correspondence: Je-Ee Ho, Department of Mechanical Engineering, National Ilan University, llan, 26047, Taiwan, Email jeho@niu.edu.tw

Received: March 13, 2017| Published: March 27, 2017

Keywords: thermoelectric chip, TB88 step-up circuit

\section{Introduction}

For the past decade, people have been dedicated to developing some clean regenerative energy without polluting environment. Among of them, thermoelectric power, generated from exhaustheat feedback, might be taken as a renewable technology. ${ }^{1,2}$ Based on various working demands, numerous typical thermoelectric chips, featured as distinguished characteristics, have been presented. ${ }^{3-7}$ While embedding it into the amplified device, a minor DC signal induced from the thermoelectric chip could be stably enhanced, which also promotes the transforming efficiency for practical purpose. ${ }^{8,9}$ However such commercial circuit- package, at present, is still too costly and confidential to be easily accessed and afforded. To improve above inadequateness, constructing a step-up loop is urgently essential, and here the booster converter of TB 88 IC, activated by low start-up voltage $0.5 \mathrm{~V}$ supplied from thermoelectric chip, guarantees a DC output of $5 \mathrm{~V}$ that meets experimental demand specified in this study (Figure 2).

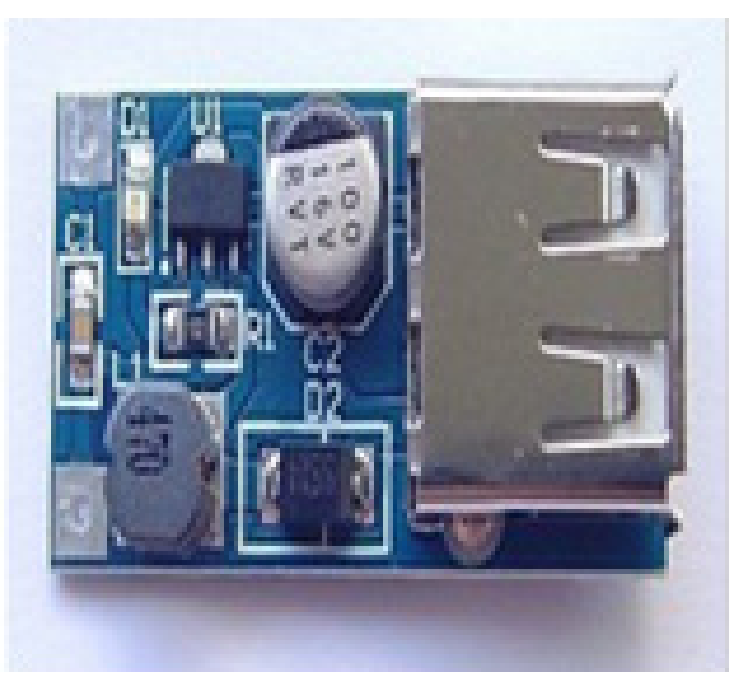

Figure I Commercial amplified device CE830I.

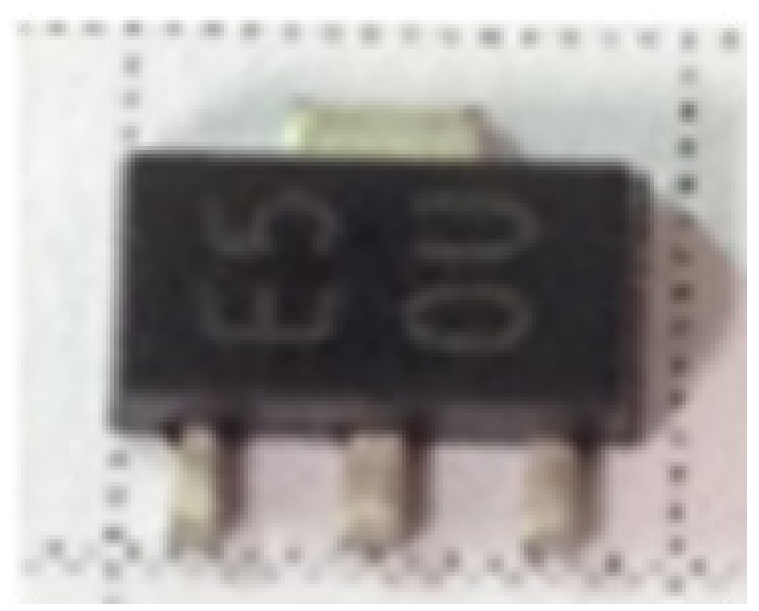

Figure 2 TB88 booster IC.

\section{Step-up circuit}

Prior to design the step-up circuit, relative theoretic analysis and fundamental principle of TB88-chip will be developed as follows:

Theoretic analysis: While the switch of transistor SW, as the block diagram of TB88 step-up circuit sketched in Figure 3, is being "ON" state, the charging to electric inductance $\mathrm{L}$ starts working and the power supplied from TEG chip will be fully absorbed. Here diode D is used to prevent the back-current from capacitor. Oppositely, a timedelay charging to capacitor, due to the non-synchronization, occurs at the energy- stored electric inductance as the switch of transistor is cut off. Such periodic charge and discharge process, the working current flowing through diode D will lift up the terminal voltage of capacitor C. Based on Kirchhoff law, power supplied from source, yielded in Eq. (1), will be fully dissipated at loaded part as well as the series of electric inductance and electric resistance. Here Vs indicates the power supplied from TEG thermoelectric chip, VO: output voltage of circuit, IL: current flowing across electric inductance, ID: output current of circuit and RL: series resistance. 


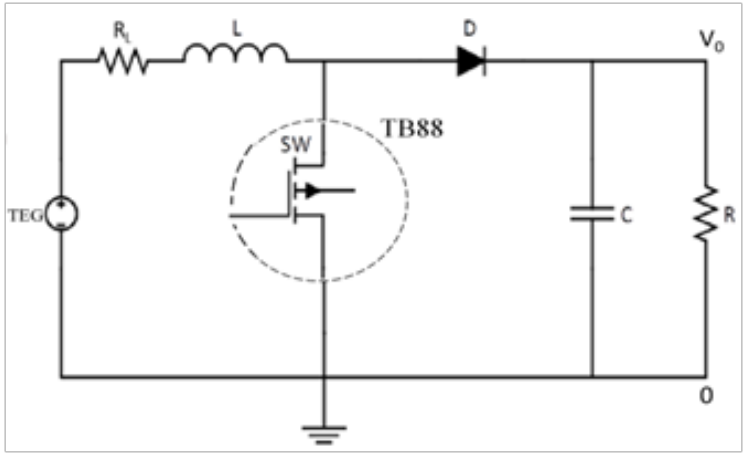

Figure 3 Block diagram of TB88 step-up circuit.

$$
V_{S} I_{L}=V_{O} I_{D}+I_{L}^{2} R_{L}
$$

As TB88-MOS being shut down, the amount of current flowing through diode and electric inductance, given in Eq. (2), is dependent on the duty cycle D of MOS, which also gives the Eq.(3), based on Ohm's law, stating the rating terminal voltage across loaded resistance. Symbol D, duty cycle, is defined as the active fraction induced by each working period of MOS.

$$
\begin{aligned}
& I_{D}=I_{L}(1-D) \\
& I_{D}=V_{O} / R
\end{aligned}
$$

Invoking Eq. (2) (3) into Eq (1), the resultant formula, after further arrangement, could be expressed in Eq. (4) and which demonstrates the magnification ration, $\mathrm{Vo} / \mathrm{Vs}$, being determined by duty cycle $\mathrm{D}$ and resistance ratio of device and electric inductance.

$$
\frac{V_{O}}{V_{S}}=\frac{(1-D)}{(1-D)^{2}+R_{L} / R}
$$

TB88-circuit: View from the pin- layout in Figure 4, the functions of oscillator, VFM control circuit, Sw switch, voltage referenced unit and error amplifier are found to be the main code of TB88 IC. Just for a low ripple of high efficiency $\mathrm{DC} / \mathrm{DC}$ converter considered in this study, only three external components including electric conductance $\mathrm{L}$, diode $\mathrm{D}$ as well as capacitor $\mathrm{C}$ is needed that might perform a special transformation for a low start-up voltage $0.5 \mathrm{~V}$ to output voltage $5 \mathrm{~V}$ while the supplied current $10 \mu \mathrm{A}$ is expected. In present experiment proposed, electric conductance $\mathrm{L}=100 \mu \mathrm{A}$, capacitor $\mathrm{C}=100 \mu \mathrm{F}$, conductance resistance $\mathrm{RL}=63 \Omega$ and duty ratio $\mathrm{D}=0.7$ are employed.

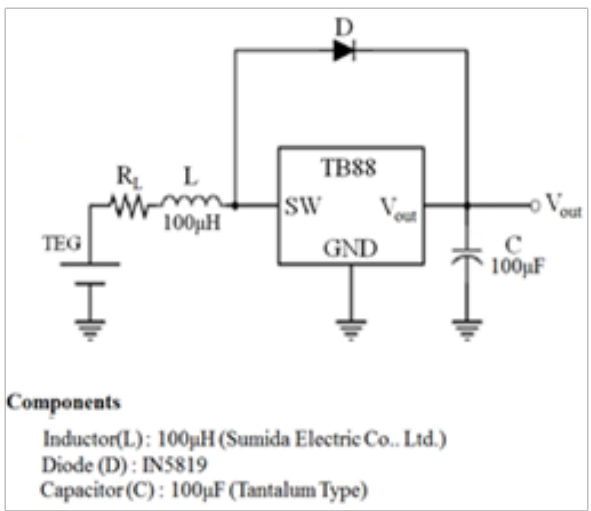

Figure 4 TB88 IC pin- layout.

\section{Experimental procedure}

\section{Self-designed step-up device}

According to the desired working demand, chip-TB88 IC with compatible electric components is selected. Using commercial package "Altium Designer summer 09" develops the layout of PCB (print circuit board see Figure 5) in advance and then install the testing components on the etched current board. After completing the stepup circuit (Figure 5), a series of test on characteristic performance, compared to the commercial product CE8301, will be undergone consecutively while the power is ready to initiate the step-up device.

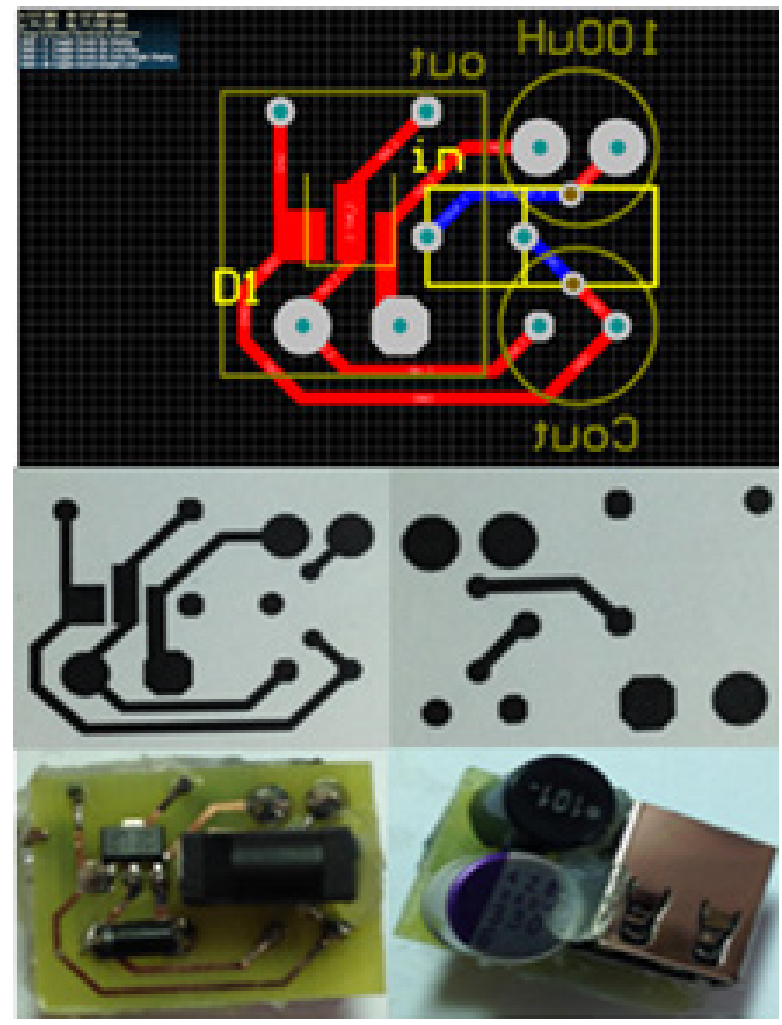

Figure 5 Self-designed step-up device.

\section{Experimental mechanism}

Accompanied with the step-up device mentioned above, an exhausted heat-generation experimental unit, shown in Figure 6, primarily consists of thermoelectric chip, heat-conduction plate, cooling fin, exhausted heat source (candle), acrylic volumetric supporter, porous base as well as desk lamp. Here the generating unit, including heat-conduction plate and cooling fin closely compacted on the bottom and top surface of thermoelectric chip, is deployed above the acrylic supporter with candle fire positioned inside, and below a porous base is mounted to induce air flow. Besides, both thermometers detecting the surficial temperature difference will be equipped on the cold and hot surface of thermoelectric chip.

\section{Experimental process}

Set up experimental mechanism with the connection of booster circuit as shown in Figure 6. Position thermocouples at the upper and lower surface of thermoelectric chip to detect temperature difference. Turn on the desk lamp after lighting up candle, and observe the illumination irradiated from lamp. During the experimental duration, 
surficial temperature difference, voltage as well as current induced from thermoelectric chip (power delivered to step-up circuit) and supplied to desk lamp (power conveyed from step-up circuit) will be recorded for each minute. then conduct a performance comparison with commercial product CE8301.

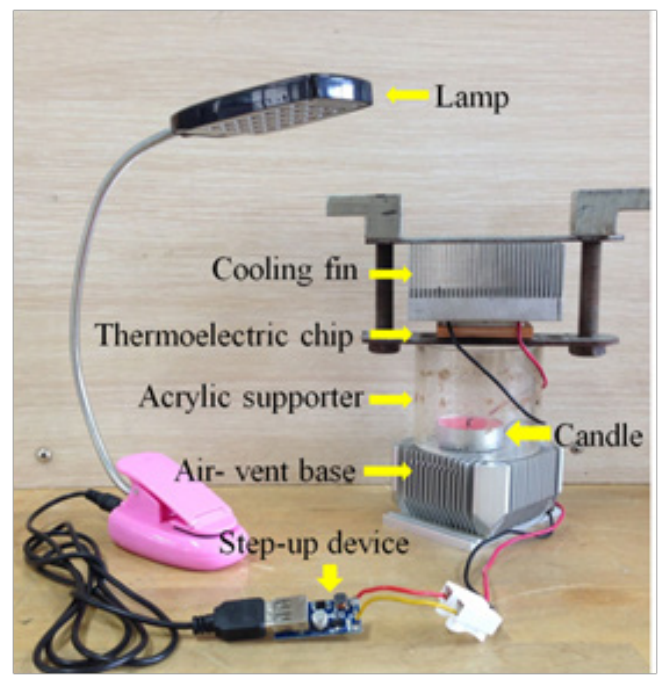

Figure 6 Experiment-mechanism photograph.

\section{Results and discussion}

Prior to our discussion, the investigation of thermoelectric performance for TEG-12703 is desired. Here electrical potential induced from the thermoelectric chip, based on the both concaved profiles distributed within the experimental duration of 15 minutes in Figure 7, is found to be closely dependent on the finite temperature difference between the colder surface, subjected to cooling fin, and hotter surface heated by candle-burning exhausted air. Here the thermal voltage Vin, $0.7 \sim 1.4 \mathrm{~V}$, related to surficial temperature difference $\Delta \mathrm{T}, 30 \sim 40^{\circ} \mathrm{C}$, could be generated from thermoelectric chip. And the maximum temperature difference $40^{\circ} \mathrm{C}$, occurring at $\mathrm{t}=6 \mathrm{~min}$, implies the heat diffused to hotter surface might be instantly conducted into environment by way of cooling fin. As thermal balance approaches at the fifteenth minute, the voltage $1.05 \mathrm{~V}$, induced from the surficial temperature difference at $30^{\circ} \mathrm{C}$, is still found too small to motivate the practical application unless the manipulation of set-up circuit is involved. To assess the suitability of self-designed TB88 IC and commercial CE8301 device embedded in the step-up circuit, the startup voltage for both should be individually investigated beforehand. While regulate power supply by term, the needed potential, in Fig. 8 , is found to be nearly $0.6 \mathrm{~V}$, i.e. voltage delivered from TEG thermoelectric chip, as discussed in Figure 7, seems to be enough to break through the energy barrier and initiate both step-up converters.

However an augmented discrepancy, in Figure 8, exists between a linear increasing from $3 \mathrm{~V}$ and stable voltage $5 \mathrm{~V}$, individually accessed from TB88 and commercial CE8301 IC, as input voltage more than $0.6 \mathrm{~V}$ is subjected. Corresponding to the induced voltage resulted in Figure 8, a more flexible operation, based on the higher circuit current of 20 130mA delivered from TEC thermoelectric chip in Figure 9, could be conducted using TB88 IC while compared to the smaller circuit current of 20 80mA conveyed with CE8301 device. Here also reveals that a special advantage might be held by TB 88 chip and the superior discrepancy, power supplied from thermoelectric chip, will be further enlarged if the thermoelectric voltage increases. Subsequently we will turn to survey the power output from the TB88 step-up circuit specified. In Figure 10, a time varying power, $10 \sim 40 \mathrm{~mW}$, as well as surficial temperature difference of thermoelectric chip, $30 \sim 40^{\circ} \mathrm{C}$, will be depicted, and the peak value of $50 \mathrm{~mW}$,delivered at temperature difference $42^{\circ} \mathrm{C}$, is primary attributed to the minimum net heat flux ,induces the maximum power generation, across the thermoelectric chip.

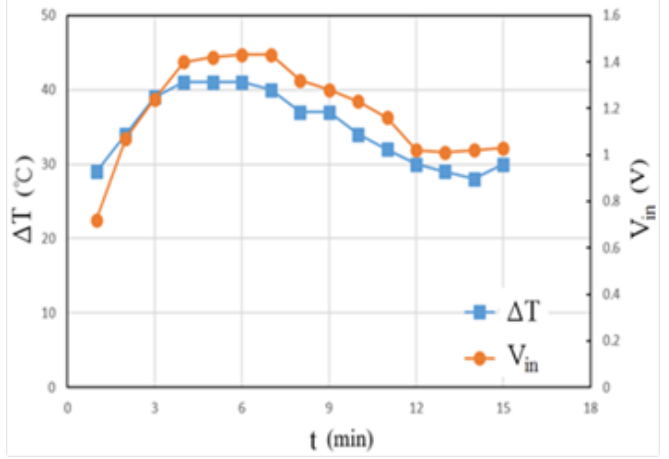

Figure 7 TEC thermoelectric volt on the surficial temperature difference $\Delta \mathrm{T}$.

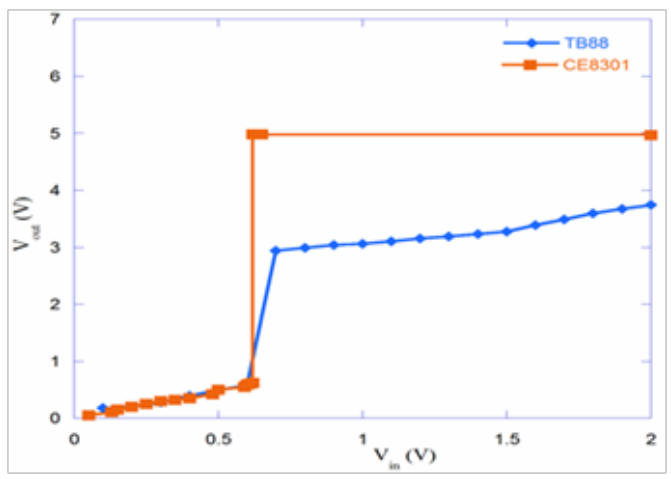

Figure 8 The induced voltage of TB88 and CE830I ICvs. thermoelectric volt.

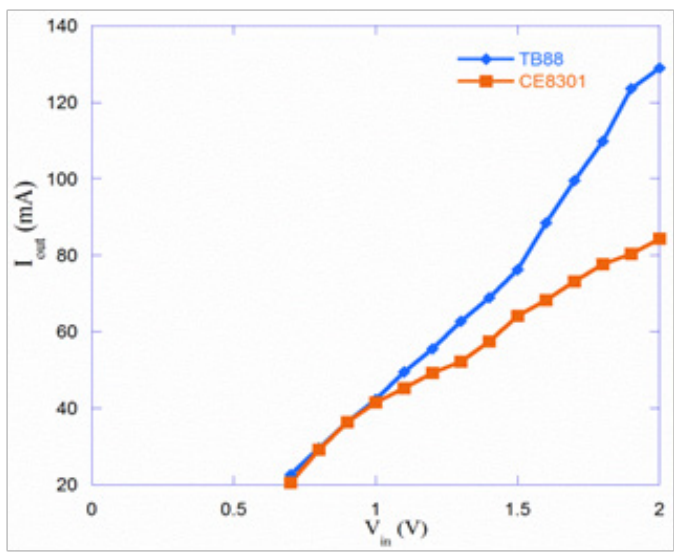

Figure 9 Comparison of thermoelectric current vs. voltage subjected to TB88 and CE830I IC.

So far the superior performance held by TB88 IC has been discussed; next our attentions will forward to evaluate the thermoelectricelectricity transforming efficiency. Based on Figures 7, Figure 9 \& Figure10. TB88- power transforming efficiency "E", defined as the ration of energy transferred from thermoelectric chip, might be estimated in Figure 11. Here a concaved profile designating 55 65\% prevails over the induced current region of $20 \sim 100 \mathrm{~mA}$, in which an 
initial increase is found to be located at region of $25 \sim 40 \mathrm{~mA}$. That attributes to the power generation from TB88 step-up circuit yielding a rapid increase $10 \sim 42 \mathrm{~mW}$ while the surficial temperature difference of thermoelectric chip $30 \sim 40^{\circ} \mathrm{C}$ is induced at the first $3 \mathrm{~min}$ in Figure 10 , and which just falls within the region of $0.7 \sim 1.3 \mathrm{~V}, 25 \sim 50 \mathrm{~mA}$ offered by TEC chip in Figures $7 \& 9$ respectively i.e. the assessed thermal power to TB88 might be estimated about $17.5 \sim 65 \mathrm{~mW}$. Thus a slow increase of transforming efficiency $55 \sim 65 \%$ might be expected in Figure 11 and an inverse intendancy of efficiency, however, will occur due to the fast growth of power provided by thermoelectric chip after the current $40 \mathrm{~mA}$ induced. Previous to enclosure our discussion, an interesting photograph, captured in Figure 12, shows that the illumination irradiated from desk lamp will be still kept for a period, no matter the blown out of candle-fire, only if the residual temperature difference exits on the surface of thermoelectric chip.

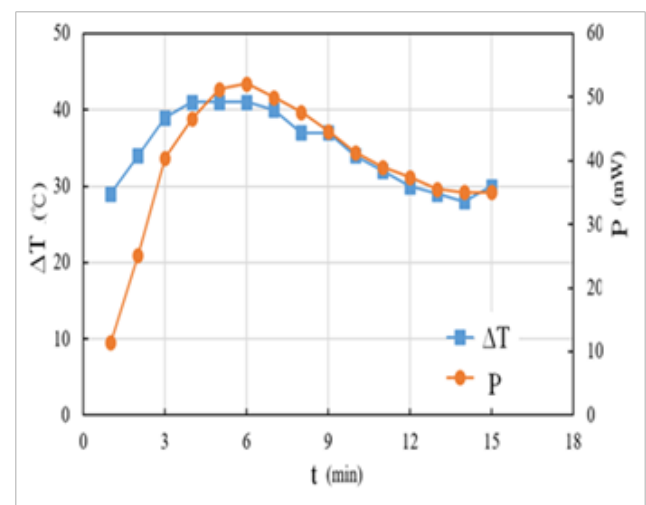

Figure 10 The power-delivery history P of TB88 step-up circuit.

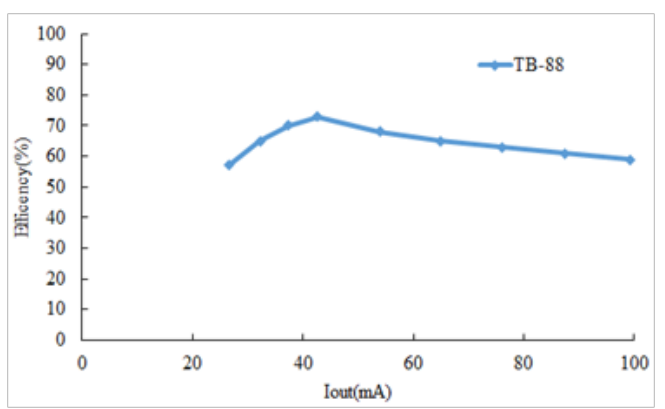

Figure I I Power transforming efficiency vs. carrying current for TB-88 chip.

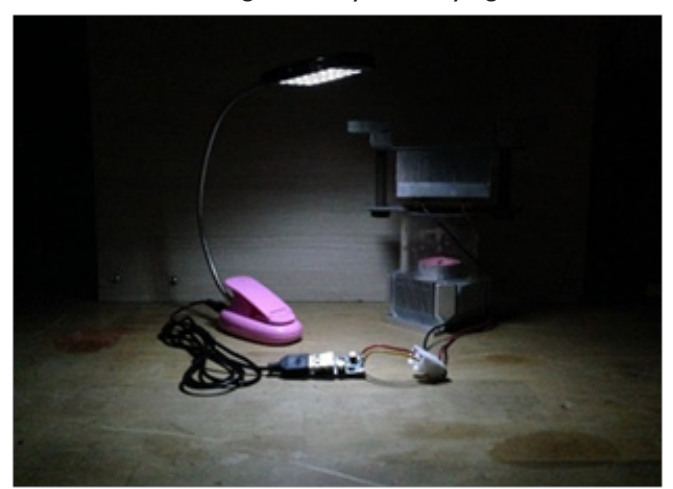

Figure I 2 The lightness irradiated from desk lamp after the candle-fire blown out.

\section{Conclusion}

Summary from above discussions, several important conclusions might be made. Firstly, directly utilize the power generated from the thermoelectric chip, based on the surficial temperature difference, seems to be impossible without the constitution of step-up circuit. Secondly, the superior performance of TB88-circuit, compared to commercial amplified device of CE8301, has been acknowledged in this article, and which not only makes the self-designed technology be significantly improved but reduces the expense in experimental budget. Finally, above skills, developed from TB88 step-up circuit for minor thermoelectric signal, could be further extended to relative practical applications such as camping lamp, auxiliary power supply from engine as well as a charging source of cell-phone.

\section{Acknowledgements}

None.

\section{Conflict of interest}

The author declares no conflict of interest.

\section{References}

1. Gou X, Xiao H, Yang S. Modeling, experimental study and optimization on low temperature waste heat thermoelectric generator system. Applied Energy. 2010;87(10):3131-3136

2. Hsiao YY, Chang WC, Chen SL. A mathematical model of thermoelectric module with applications on waste heat recovery from automobile engine. Applied Energy. 2010;35(3):1447-1454.

3. Chen L, Sun F, Wu C. Thermoelectric-generator with linear phenomenological heat-transfer law. Applied Energy. 2005;81(4):358 364 .

4. Khattab NM, Shenawy ET El. Optimal operation of thermoelectric cooler driven by solar thermoelectric generator. Applied Energy Conversion and Management. 2006;47:407-426.

5. Chen L, Gong J, Sun F, et al. Effect of heat transfer on the performance of thermoelectric generators. Applied International Journal of Thermal Science. 2001;41(1):95-99.

6. Chen L, Sun F, Wu C. Performance optimization of a twostage semiconductor thermoelectric-generator. Applied Energy. 2005;82(4):300-312.

7. Cheng JW, Tsai HL. Waste-heat recovery from an internal combustion engine for reuse in a vehicle air-conditioning system. Applied Journal of Science and Engineering Technology. 2010;6(3):23-28.

8. Chen RCH, Lesmana T, Chung DWY, et al. Autonomous wearable ECG readout circuit using body heat energy harvesting. Applied Department of Electronic Engineering, Taiwan: Chung Yuan Christian University; 2013.

9. Carlson E, Strunz K, Otis B. 20mV input boost converter for thermoelectric energy harvesting. Applied IEEE symposium VLSI Circuits Dig Tech Papers; 2009. p. 162-163. 